\title{
Shear thinning and thixotropy of HMHEC and HEC water solutions
}

\author{
A. Maestro, ${ }^{\text {a) }}$ C. González, and J. M. Gutiérrez \\ Chemical Engineering and Material Science Department, Barcelona University, \\ Martí i Franquès 1, E-08028 Barcelona, Spain
}

(Received 4 June 2002; final revision received 29 August 2002)

\begin{abstract}
Synopsis
Steady state viscosity and thixotropy of hydrophobically modified hydroxyethyl cellulose (HMHEC) and nonassociative cellulose water solutions are studied. Although all the samples are shear thinning, only the HMHEC is thixotropic, since the migration of hydrophobes to micelles is controlled by diffusion. The Cross model fits steady state curves. The Mewis model, a phenomenological model that proposes that the rate of change of viscosity when the shear rate is suddenly changed is related to the difference between the steady state and current values of viscosity raised to an exponent, fits structure construction experiments when the exponent, $n$, is estimated to be around 2. The Newtonian assumption used by Mewis cannot be used here, however. This seems to be related to the fact that the thickening is due to bridged micelle formation, which is a slow process, and also to topological constraints and entanglements, which are rapid processes. The kinetic parameter was redefined to $k_{n}$ in order to make it independent of initial conditions. So, $k_{n}$ depends only on how the shear affects the structure. $k_{n}$ reaches a plateau at shear rates too low to produce structure destruction and decreases at higher shear rates. (C) 2002 The Society of Rheology. [DOI: 10.1122/1.1516789]
\end{abstract}

\section{INTRODUCTION}

Latex paints require the addition of thickeners in order to attain a proper rheology. Thickeners traditionally used were water-soluble derivatives of cellulose, such as hydroxyethyl celluloses of high molecular weight (HEC) [Wetzel et al. (1996)]. These derivatives thicken the medium because of chain entanglements between the long polymer molecules, which are solvated and extended in an aqueous medium [McCormick and Johnson (1989); Emmons and Travis (1978)]. Coatings thickened with HECs have high resistance to sagging, but poor leveling [Seneker and Glass (1996); Schwab (1986); Croll and Kleinlein (1986)]. To solve this problem, in the last few years these polymers have been substituted in some applications by associative polymers (APs) [Wetzel et al. (1996); Yekta et al. (1993); Hoy and Hoy (1984)], which consist of a hydrophilic backbone to which hydrophobic groups have been attached so that each molecule has two or more hydrophobes [Tarng et al. (1996); Sau and Landoll (1989)] that are randomly distributed along the polymer chain [Xu et al. (1997)] or are present as terminal groups [Tam et al. (1998)]. In an aqueous medium, these groups form aggregates that are similar to surfactant micelles. Hydrophobes of the same molecule can be joined to the same micelle, forming a loop [Yekta et al. (1995)]. Then flower-like independent micelles

\footnotetext{
a) Author to whom all correspondence should be addressed; electronic mail: maestro@angel.qui.ub.es
} 
form. But these can also aggregate to different micelles. When this happens micelles are bridged by hydrophilic backbones and a three-dimensional network forms that enhances the viscosity of the medium. Under shear conditions, bridges are stretched, hydrophobes are forced to leave their micelles, and a bridge-to-loop transition takes place that weakens the network, resulting in a decrease of viscosity.

Linear viscoelasticity of these polymers has been extensively studied [Maestro et al. (2002); Tam et al. (1998); Svanholm et al. (1997); Xu et al. (1996); Groot and Agterof (1995); Annable and Buscall (1993); Yekta et al. (1993); Kaczmarski and Glass (1993); Tanaka et al. (1992); Glass et al. (1991); Karunasena et al. (1989)] because it gives information about the thickening mechanism. However, it is not useful for understanding behavior during painting because it takes place out of the linear range, since changes in the structure occur. In fact, the dependence of viscosity on shear (steady state curve), and the kinetics of the viscosity variation when the shear rate or the shear stress is modified (thixotropy), are the determinants in the handling of the paint and quality of the final film. Thus, they seem interesting enough to be quantified.

Most associative polymers are found to be shear dependent [Zhang (2001); Reuvers (1999); Svanholm et al. (1997); Xu et al. (1996); Kästner (2001); Sau and Landoll (1989); Goodwin et al. (1989)]. A plateau region followed by shear thickening and shear thinning regions at higher shear rates is observed for HEUR solutions [Jenkins et al. (1996); Tarng et al. (1996); Svanholm et al. (1997); Annable and Buscall (1993); Xu et al. (1996)]. The shear thickening behavior at intermediate shear rates is attributed to a change from intramolecular to intermolecular association as molecules become elongated under shear [Jenkins et al. (1991); Witten and Cohen (1985)]. Shear thinning is also observed with HMHEC solutions [Svanholm et al. (1997); Sau and Landoll (1989); Goodwin et al. (1989)]. Goodwin and Hughes (1997) proposed the Cross model [Cross (1965)] or the Papir model [Papir and Krieger (1970)] for the fitting of viscosity curves. These models do not fit shear thickening behavior.

Besides being shear dependent, a large number of associative polymers are thixotropic [Kästner (2001); Vittadello and Biggs (1998); Tarng and Glass (1995); Kästner et al. (1996); Kroon (1993)], although their thixotropy has not been studied in a quantitative way, nor has systematic experimentation been developed. Some interesting models have been created that try to predict thixotropic behavior of fluids. They fall into four groups: first, there are those models that use a very general description of microstructure described by a numerical value of a scalar parameter $\lambda$, which can vary between 0 (completely destroyed structure) and 1 (completely formed structure). Some authors of this group are Tiu and Boger (1974), Alessandrini et al. (1982), De Kee et al. (1983), and Baravian et al. (1996). Second are those authors who attempt some direct description of the temporal change of the microstructure such as, for example, the number of bonds or the size of the aggregates, for instance, Denny and Brodkey (1962) or Lapasin et al. (1996). Third, there are authors that use the viscosity-time data itself on which to base a theory, such as Fredrickson (1970) or Mewis and Schryvers (1996) reported by Barnes (1997). Finally, a number of authors use viscoelastic models, termed "thixoelastic" by some. They introduce some modifications in the linear viscoelasticity expressions in order to describe structural changes and obtain nonlinear viscoelasticity expressions. Some of the authors in this last group are Acierno et al. (1976), Soong and Shen (1979), Bautista et al. (1999), and Quemada (1999).

None of the models we know of has been used for a description of the thixotropy of the associative polymers used in paints. In the present article, the steady state curves and thixotropy of HMHEC are studied and compared with HECs of several molecular weights. A simple model is developed for an initial quantification of thixotropy. 
TABLE I. Thickeners used in the experiments.

\begin{tabular}{lcccc}
\hline \hline & & \multicolumn{2}{c}{$M_{W}$} & \\
& Acronym & (gmol & \\
& HEC9 & 90000 & 1.5 & 2.5 \\
\hline $\begin{array}{l}\text { (2-hydroxyethyl)cellulose } \\
\text { (2-hydroxyethyl)cellulose }\end{array}$ & HEC130 & 1300000 & 1.5 & 2.5 \\
(2-hydroxyethyl)cellulose, hydrophobically modified with & HMHEC & 560000 & 2.0 & $2.7-3.4$ \\
hexadecyl groups & & & & \\
\hline \hline
\end{tabular}

\section{EXPERIMENT}

\section{A. Materials and preparation}

All compounds were obtained from Aldrich. Molecular weight $M_{w}$, molar substitution $M S$, and degree of substitution $D S$ of cellulose derivatives are summarized in Table I.

The celluloses were received as a solid powder and were used as supplied. De-ionized water further purified through a Millipore MilliQ purification system was used to prepare solutions. Water was stirred and heated to $40{ }^{\circ} \mathrm{C}$, and polymer was gradually added. Stirring was maintained for $2 \mathrm{~h}$ and solutions were stored for at least $24 \mathrm{~h}$ before use to assure complete hydration. When butoxy ethanol was used, a 20\% butoxy ethanol-80\% water solution was prepared before the addition of the thickener. Solutions were used during a 7-day period in order to avoid undesirable effects produced by enzymatic degradation.

\section{B. Rheological measurements}

All rheological measurements were performed with a Haake RS150 rheometer. This rheometer can work either as a controlled shear rate or a controlled shear stress rheometer. The controlled shear rate mode was chosen. Two different sensors were used, depending on the range of viscosity of the solutions, so as to ensure adequate sensitivity. A $60 \mathrm{~mm}$ diam double cone and $1^{\circ}$ cone angle was used for solutions with small viscosity, and a cone-and-plate sensor with a $60 \mathrm{~mm}$ diameter and $4^{\circ}$ cone angle was used for the other solutions. Some measurements were carried out with both sensors, and results were compared to assure they did not influence the experimental data. The temperature was controlled, with a deviation less than $\pm 0.2{ }^{\circ} \mathrm{C}$.

Steady state curves were obtained for a range of shear rates between 0.07 and $500 \mathrm{~s}^{-1}$.

Evolution of the viscosity over time when the shear rate changes suddenly from one value to another was measured. The initial shear rate was maintained until the steady state was reached, and then it was changed to the final shear rate, which was maintained for 15 min. Data of viscosity versus time were taken throughout the experiment.

\section{RESULTS AND DISCUSSION}

\section{A. Viscosity as a function of shear rate (steady state curve)}

Figure 1 shows the steady state viscosity as a function of shear rate for HEC and HMHEC solutions. For comparative purposes, we present the concentrations where similar low shear viscosities $\eta_{0}$ were obtained. For a $\eta_{0}$ around $1.3 \mathrm{Pas}, 10 \%$ HEC9 is needed, but only $0.75 \%$ HEC 130 or $0.5 \%$ HMHEC. As expected, the thickening efficiency of HEC increases with the molecular weight due to the presence of more interactions and entanglements. Hydrophobic modification of HEC increases efficiency because 


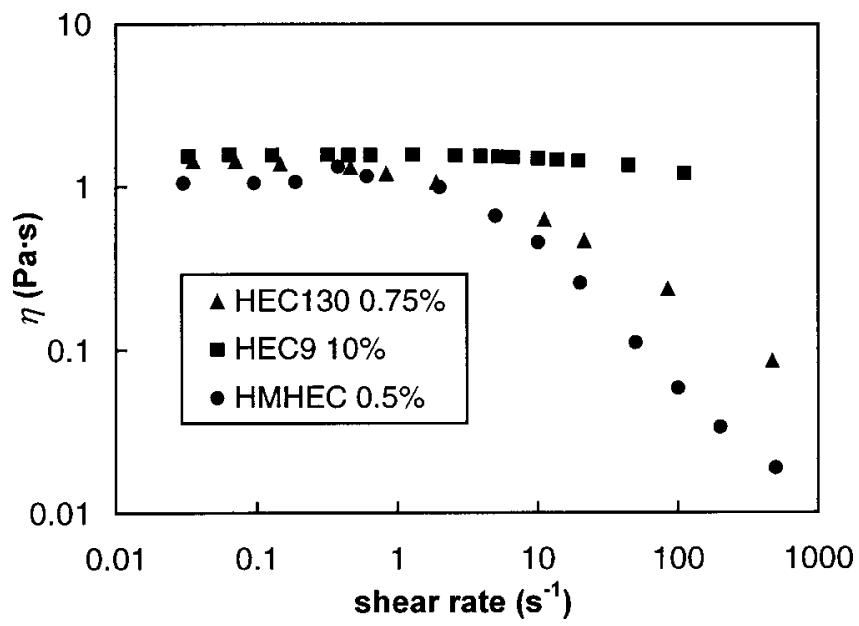

FIG. 1. Steady state curves for several cellulosic derivatives. Temperature $=20^{\circ} \mathrm{C}$.

of the formation of a three-dimensional micellar network. HMHEC solution has the higher shear thinning behavior because of the shear-induced transitions from inter- to intramolecular association.

The influence of temperature and concentration on the steady state curve of HMHEC was studied. Figure 2 shows curves at different concentrations, and Fig. 3 at different temperatures. The concentration increases viscosity over all the shear rate range because of the presence of a large number of micelle-like aggregates, which favors the formation of bridges and enhances the network. Shear thinning behavior is observed for all concentrations studied. Only at the lower concentration is a previous shear thickening region observed. Tam et al. (1998) attributed the shear thickening behavior observed in HEUR solutions to shear-induced loop-to-bridge transitions that increase the fraction of micelles included in the mechanically active network and increase the number of bridges. At higher concentrations of HMHEC, this shear thickening region is not observed, probably because when the fraction of micelles is high enough micelles are close together and the

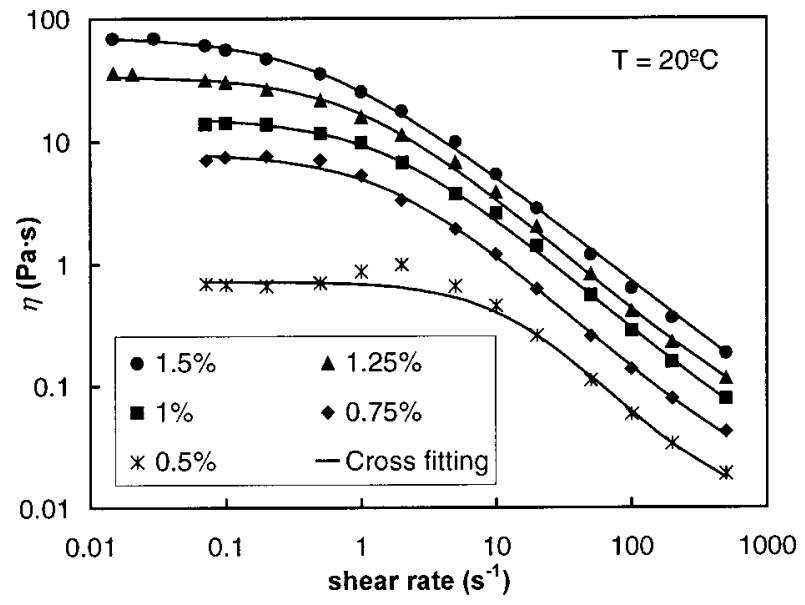

FIG. 2. Steady state curves for HMHEC aqueous solutions at $20^{\circ} \mathrm{C}$ and several concentrations. 


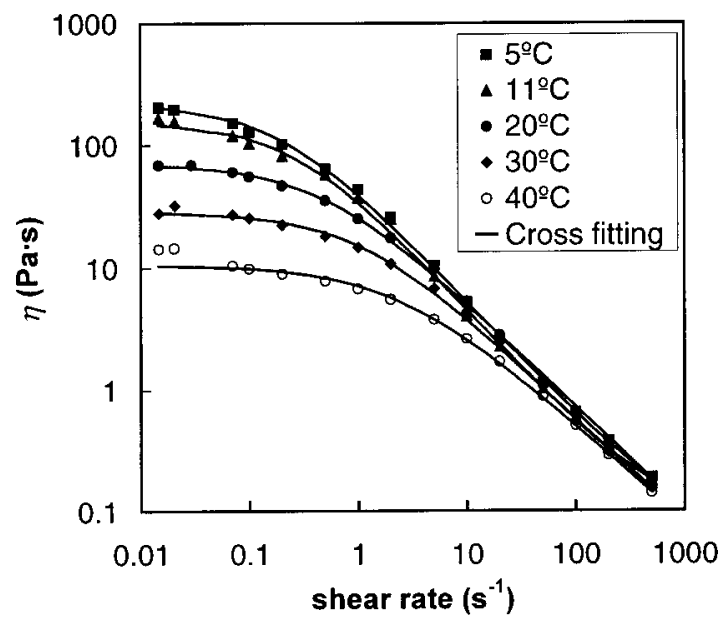

FIG. 3. Steady state curves for HMHEC $1.5 \%$ at several temperatures.

number of bridges is not limited by the distance between them. As a result, chain elongation produced by intermediate shear, which favors hydrophobic interactions at low concentrations, does not have much influence on the number of bridges when the concentration is high.

The experimental data shown in Figs. 2 and 3 were fitted through the Cross model [Eq. (1)], where $\eta_{0}$ and $\eta_{\infty}$ are the low and high shear viscosities, respectively. The exponent $m$ is found to be around 0.9. As shown, the fitting is quite good, except at $0.5 \%$, because the shear thickening behavior cannot be described by the Cross model,

$$
\eta(\dot{\gamma})=\eta_{\infty}+\frac{\eta_{0}-\eta_{\infty}}{1+\left(\dot{\gamma} / \dot{\gamma}_{c}\right)^{m}} .
$$

The high shear viscosity cannot be known accurately because of limitations of the measuring range and possible hydrodynamic instabilities at very high shear rates, although the results were very reproducible and no oscillation or evidence of instability was observed for these shear rates. Figure 3 shows that the low shear viscosity $\eta_{0}$ decreases with temperature $T$, but viscosity tends to be independent of $T$ at high shear rates. An Arrhenius dependence on the temperature was found for $\eta_{0}$, in agreement with other authors [Annable and Buscall (1993)]. The potential barrier to disengagement of a chain end from a junction point, $E_{m}$, which may be equated to the binding energy of the hydrophobe to its micelle [Tam et al. (1998)], is determined to have a value of $E_{m}$ $=72 \mathrm{~kJ} / \mathrm{mol}$, similar to that found by Annable for HEUR polymers. At higher shear rates the Arrhenius dependence is lost. While at low shear, in the Newtonian range, the associated structure and, as a consequence, the viscosity, depend on thermal motion, at higher shear rates thermal dependence is negligible because it is masked by shear effects. Thus, viscosity tends to be independent of the temperature.

\section{B. Thixotropy}

\section{Thixotropy of HEC and HMHEC}

The evolution of viscosity over time when the shear rate changes suddenly was studied for solutions of HECs and HMHEC. An example of the results is shown in Fig. 4, where it can be seen that the viscosity of the HECs studied increases and quickly reaches the 


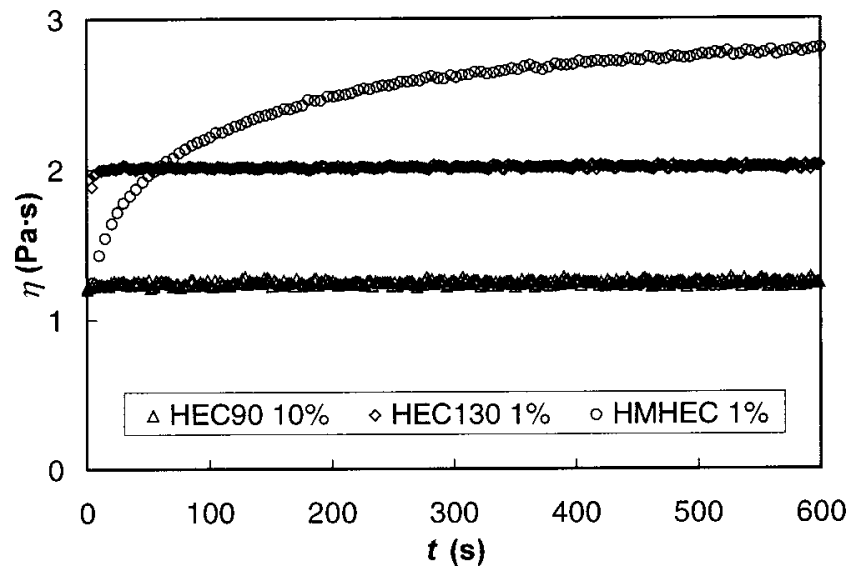

FIG. 4. Viscosity $(\eta)$ vs time $(t)$ when the shear rate is changed suddenly from 2000 to $8 \mathrm{~s}^{-1}$. Temperature $=20^{\circ} \mathrm{C}$.

steady state viscosity corresponding to the new shear rate. The same happens at all temperatures and concentrations tested. So, thixotropy of these HECs is so fast that the kinetics cannot be quantified. In contrast, the increase of viscosity for the HMHEC solution is gradual enough to be measured, so that a significant thixotropic behavior is observed. These differences between HEC and HMHEC are in agreement with Kästner studies $(1996,2001)$. We attribute them to the fact that the thickening mechanism of HECs is due to topological constraints and entanglements between the long chains of polymer, which are fast processes. On the other hand, although topological constraints and entanglements also exist for the HMHEC water solutions, the main thickening mechanism is due to hydrophobic interactions. So, the recovery of viscosity requires the migration of hydrophobes through the medium towards the micelles, which is a slow diffusion-controlled process. It can be concluded that the HEC water solutions studied do not have significant thixotropy at the concentrations and temperatures tested. The thixotropic behavior of HMHEC water solutions is studied in Sec. III B2.

\section{Model used for HMHEC}

Experiments were carried out in which the shear rate was suddenly changed from a low value to higher ones (structure destruction experiments), and from a high value to lower ones (structure construction experiments). Figure 5 shows the results obtained when the shear rate was changed from $0.1 \mathrm{~s}^{-1}$ to several higher values. Viscosity is seen to decrease over time and tends to move toward the new equilibrium viscosity because of the structure destruction produced by shear. An overshoot is observed at short times; this indicates the presence of elasticity [Bautista et al. (1999); Barnes (1997)]. This is supported by the fact that HMHEC solutions were found to be viscoelastic in publications where linear viscoelasticity was studied [Maestro et al. (2002); Karlson et al. (2000); Svanholm et al. (1997); Brown (1994); Sau and Landoll (1989); Goodwin et al. (1989); Landoll (1982)]. Although this overshoot is not so evident at $5 \mathrm{~s}^{-1}$, and cannot be observed at $50 \mathrm{~s}^{-1}$ or higher shear rates, it is only due to partial masking of the effect produced by the shear. As a result, if some model were to be found for the description of thixotropic behavior, elasticity should be included. Some authors have successfully combined thixotropy and elasticity [Bautista et al. (1999); Quemada (1999); Barnes (1997); Mewis (1979); Acierno et al. (1976)]. Some, like Quemada or Bautista, find relatively 


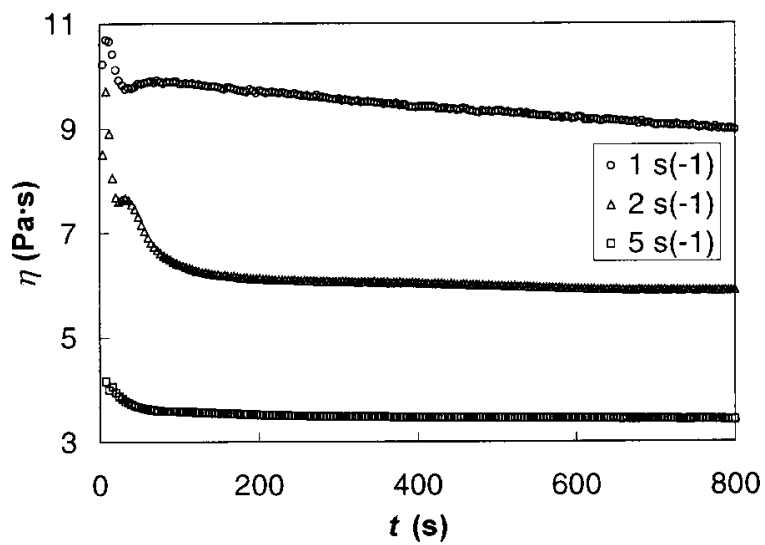

FIG. 5. Viscosity vs time when the shear rate is changed suddenly from $0.1 \mathrm{~s}^{-1}$ to several higher shear rates. HMHEC $1 \%$ and $20{ }^{\circ} \mathrm{C}$.

simple models using a simple Maxwell equation for the description of linear viscoelasticity. However, when a generalized Maxwell model is used, the models obtained remain rather complex and thus difficult to use in practice. Linear viscoelastic characterization of HMHEC solutions indicates that it is not a simple Maxwell fluid [Karlson et al. (2000); Svanholm et al. (1997)], but it has a relaxation time spectrum that is the result of two relaxation processes: a long relaxation process with a logarithmic distribution of relaxation times, related to the lifetime of hydrophobic junctions, plus a relaxation process related to rapid relaxation of topological constraints [Maestro et al. (2002)]. So, the fitting of destruction experiments should require the development of a very complex model. This is not the purpose of this article, but it is the development of some simple model that could quantify the change of viscosity over time in a simple way with only a few parameters. Figure 6 shows an example of construction experiments where the shear rate is changed from an initial shear rate $\dot{\gamma}_{0}=2000 \mathrm{~s}^{-1}$ to several smaller shear rates

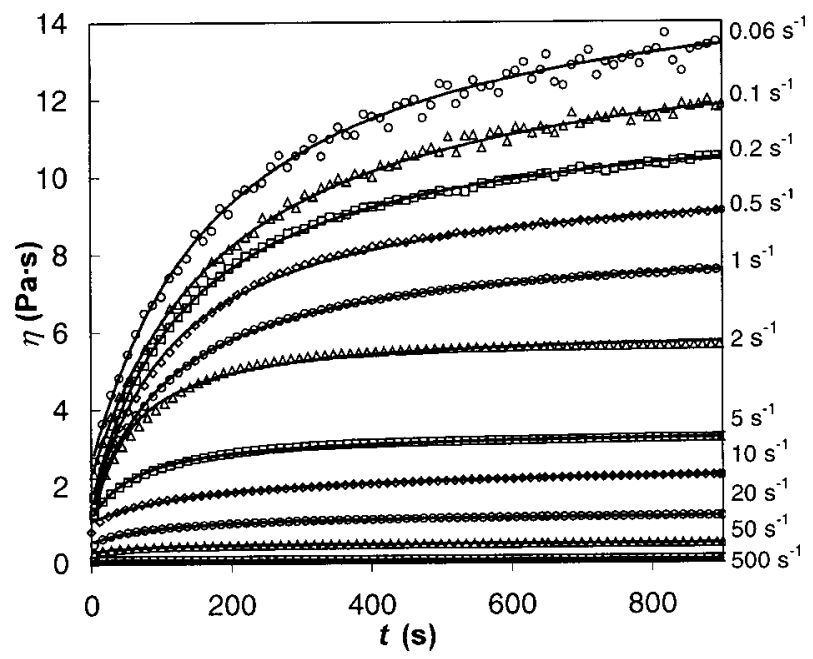

FIG. 6. Viscosity vs time when the shear rate is changed suddenly from $2000 \mathrm{~s}^{-1}$ to several lower shear rates. Fittings are shown as a continuous line. HMHEC $1 \% ; T=20^{\circ} \mathrm{C}$. 
$\dot{\gamma}_{1}$. Although, of course, these curves are also influenced by elasticity, their shape seems to indicate that a single kinetic constant could be used that would include pure thixotropy and elasticity. This seems interesting for initial quantification of the thixotropy of an associative polymer.

A phenomenological model devised by Mewis and Schryvers [Barnes (1997)] was used to fit results. It proposes that the rate of change of viscosity when the shear rate is suddenly changed from an initial shear rate $\dot{\gamma}_{0}$ (at the steady state) to a new one $\dot{\gamma}_{1}$ is related to the difference between the steady state and current values of viscosity:

$$
\frac{d \eta}{d t}=k\left[\eta_{e}\left(\dot{\gamma}_{1}\right)-\eta\right]^{n}
$$

This integrates as

$$
\eta=\eta_{e}-\left(\eta_{e}-\eta_{i}\right)\left[(n-1) k t\left(\eta_{e}-\eta_{i}\right)^{n-1}+1\right]^{1 /(1-n)},
$$

where $\eta_{e}$ is the equilibrium viscosity at a given shear rate $\dot{\gamma}_{1}, \eta_{i}$ is the initial viscosity for this new shear rate $\dot{\gamma}_{1}$, and $n$ is the kinetics order. Mewis and Schryvers then made the Newtonian assumption $\eta_{i}\left(\dot{\gamma}_{1}\right)=\eta_{e}\left(\dot{\gamma}_{0}\right)$, which makes use of the equation simpler because it is easier to measure the viscosity of the initial steady state condition. This assumes that the viscosity at the end of the initial steady state period is the same as that at the beginning of the new shear rate test, i.e., the system is Newtonian between these conditions and viscosity is only a function of the quantity of structure formed and not a function of both the structure and shear rate. However, as Mewis points out, this is only true for high shear rates.

Figure 6 shows some examples of fitting experimental data using the Mewis and Schryvers model, but with some modifications. First of all, the kinetics order $n$ was found to be $n \cong 2$. Second, the model was unable to fit results if the Newtonian assumption used by Mewis was made. So, $\eta_{i}$ was used as a free parameter. Then, the equation used to fit experimental data was

$$
\eta=\eta_{e}+\frac{1}{1 /\left(\eta_{i}-\eta_{e}\right)-k t} .
$$

Three parameters are used: the equilibrium viscosity at the new shear rate, $\eta_{e}$; the initial viscosity at the new shear rate, $\eta_{i}$; and the kinetic constant, $k$.

\section{Shear rate dependence of the fitting parameters}

Experiments at several initial and final shear rates were performed. The dependence of the initial viscosity $\eta_{i}$ on the initial $\left(\dot{\gamma}_{0}\right)$ and the final $\left(\dot{\gamma}_{1}\right)$ shear rate is shown in Fig. 7. As expected, $\eta_{i}$ decreases with $\dot{\gamma}_{0}$, since the transient network is destroyed under shear and, at time $t=0$, when shear rate has just been changed, the intramolecular to intermolecular balance corresponding to the initial state still remains. However, $\eta_{i}$ also decreases with $\dot{\gamma}_{1}$ for the same $\dot{\gamma}_{0}$, indicating that the Newtonian assumption proposed by Mewis cannot be used here. This is attributed to the fact that topological constraints and chain entanglements, which are rapid processes, are one of the factors that determine the viscosity of HMHEC solutions, besides bridged micelle formation. Figure 8(a) shows the steady state viscosity of HMHEC solutions when $20 \%$ of butoxy ethanol was added in order to prevent micelle formation. It can be seen that this solution is also shear thinning, although, of course, to a much smaller extent than HMHEC, indicating that chain interactions or entanglements contribute to the thickening besides hydrophobic interactions. For the same solution, Fig. 8(b) shows time curves when the shear rate changes from an 


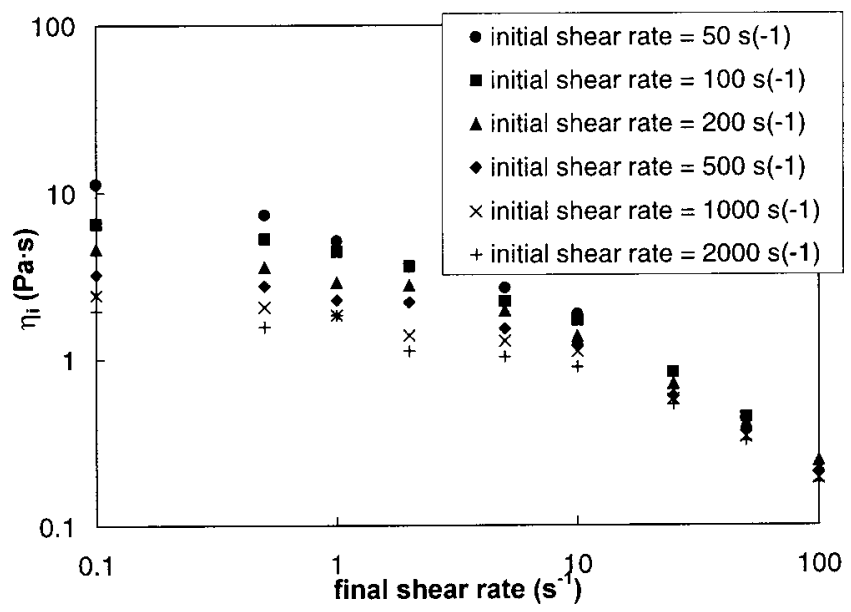

FIG. 7. Initial viscosity vs final shear rate at several initial shear rates. HMHEC $1 \% ; T=20^{\circ} \mathrm{C}$.

initial shear rate $\dot{\gamma}_{0}=2000 \mathrm{~s}^{-1}$ to several smaller shear rates $\dot{\gamma}_{1}$. It can be seen that equilibrium viscosity is quickly reached and no significant thixotropy is observed. So, besides the slow process of micelle formation, the HMHEC solutions are characterized by a rapid relaxation process that is very likely responsible for the invalidity of the Mewis Newtonian assumption.

The equilibrium viscosity $\eta_{e}$, as expected, is independent of $\dot{\gamma}_{0}$ and shows shear thinning behavior with $\dot{\gamma}_{1}$. The results coincide with the steady state curves found through shear rate sweeps and shown in Fig. 2. This indicates that $\eta_{e}$ is correctly estimated by the model proposed.

Figure 9 shows that the kinetic constant $k$ depends on $\dot{\gamma}_{1}$, and also on $\dot{\gamma}_{0}$, i.e., it depends on the initial conditions. This constant was substituted for a new kinetic constant $k_{n}$ defined as

$$
k_{n}=k\left(\eta_{e}-\eta_{i}\right)^{2} .
$$

Equations (2) and (4) can be rewritten as follows:
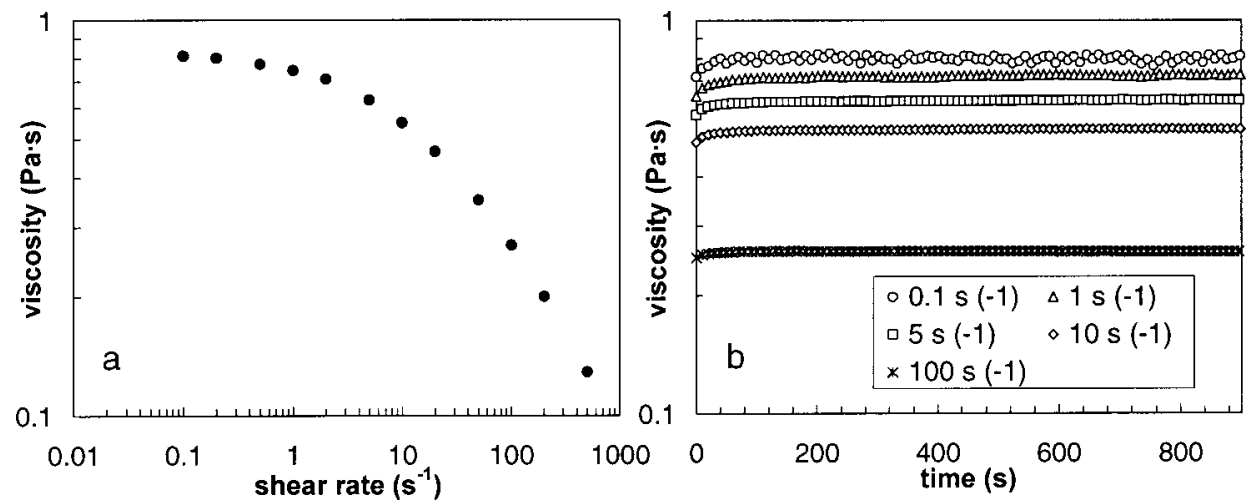

FIG. 8. Rheological measurements of $1.5 \%$ HMHEC aqueous solutions with $20 \%$ of butoxy ethanol; $T=20^{\circ} \mathrm{C}$. (a) Steady state curves; (b) viscosity vs time when the shear rate is changed suddenly from 2000 $\mathrm{s}^{-1}$ to several lower shear rates. 


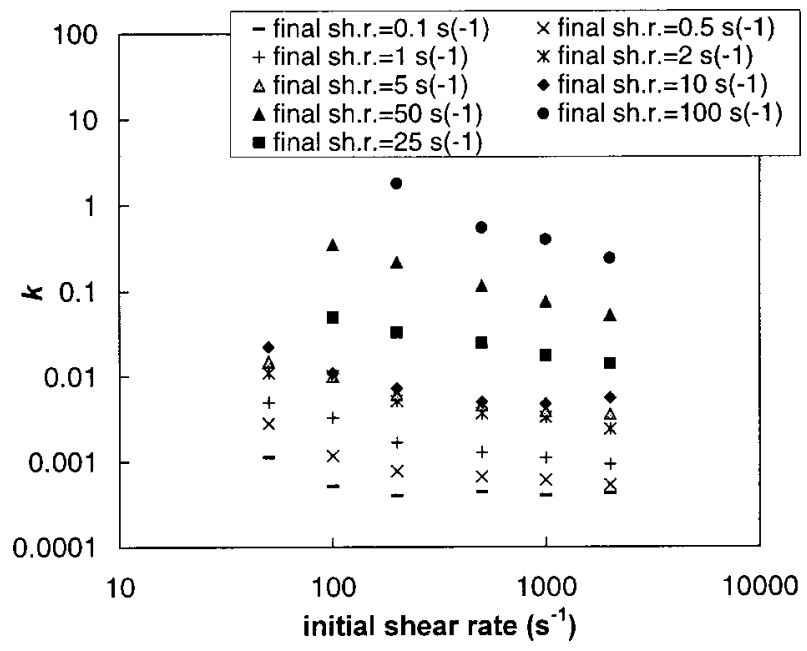

FIG. 9. Kinetic constant $(k)$ vs initial shear rate at several final shear rates. HMHEC $1 \% ; T=20^{\circ} \mathrm{C}$.

$$
\begin{gathered}
\frac{d \eta}{d t}=k_{n}\left[\frac{\eta_{e}(\dot{\gamma})-\eta}{\eta_{i}-\eta_{e}}\right]^{2} \\
\eta=\eta_{e}+\frac{\eta_{i}-\eta_{e}}{1-\left[k_{n} /\left(\eta_{i}-\eta_{e}\right)\right] t} .
\end{gathered}
$$

Figure 10 shows the dependence of $k_{n}$ on $\dot{\gamma}_{1}$ for several $\dot{\gamma}_{0}$. It can be observed that $k_{n}$ is independent of $\dot{\gamma}_{0}$. On the other hand, $k_{n}$ decreases with $\dot{\gamma}_{1}$ for high shear rates, but takes a constant value at lower ones. So, this curve qualitatively has the same shape as the steady state curve (Fig. 2). In the range of high shear rates, the shear rate greatly influences the structure. Here, $k_{n}$ increases when the shear rate is decreased. On the other hand, in the range of low shear rates, where the shear does not affect the structure and, as a consequence, a plateau is observed for the equilibrium viscosity, $k_{n}$ becomes independent of the shear rate. It now appears that $k_{n}$ only depends on how the shear rate affects the structure.

\section{CONCLUSIONS}

The HEC and the HMHEC water solutions tested present shear thinning behavior. Only the HMHEC presents thixotropy, since transitions from intra- to intermolecular associations are limited by diffusion.

Steady state curves obtained with HMHEC can be fitted through the Cross model for concentrations higher than $0.75 \%$. For lower concentrations, shear thickening behavior is observed at intermediate shear rates, due to shear-induced transitions from intra-to intermolecular associations.

As HMHEC is a viscoelastic fluid, elastic effects are observed coupled to thixotropy. The Mewis and Schryvers model, with some modifications, properly fits structure construction experiments. However, the Newtonian assumption $\eta_{i}\left(\dot{\gamma}_{1}\right)=\eta_{e}\left(\dot{\gamma}_{0}\right)$ used by Mewis cannot be made for this case since, although the main thickening mechanism is due to hydrophobic interactions, viscosity is also influenced by rapid chain interactions and entanglements. 


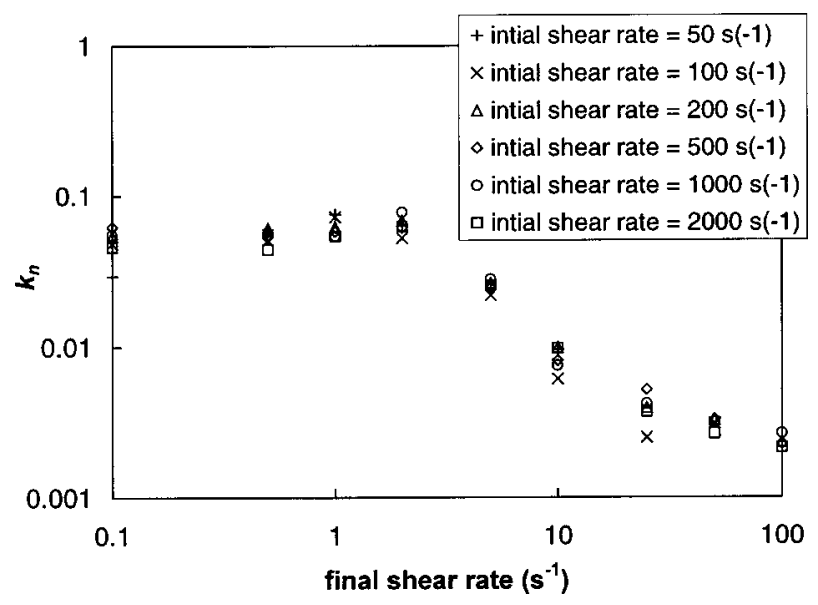

FIG. 10. Kinetic constant $\left(k_{n}\right)$ vs final shear rate at several initial shear rates. HMHEC $1 \% ; T=20^{\circ} \mathrm{C}$.

The kinetic parameter $k$ can be redefined to $k_{n}=k\left(\eta_{e}-\eta_{i}\right)^{2}$. This parameter is independent of the initial conditions and only depends on how the shear rate affects the structure. Then, $k_{n}$ decreases with shear at the higher shear rates and takes a constant value at shear rates too low to affect the structure.

\section{References}

Acierno, D., F. P. La Mantia, G. Marrucci, and G. Titomanlio, "A non-linear viscoelastic model with structuredependent relaxation times," J. Non-Newtonian Fluid Mech. 1, 125-146 (1976).

Alessandrini, A., R. Lapasin, and F. Sturzi, "The kinetics of thixotropic behavior in clay/kaolin aqueous solutions," Chem. Eng. Commun. 17, 13-22 (1982).

Annable, T., and R. Buscall, "The rheology of solutions of associating polymers: Comparison of experimental behavior with transient network theory," J. Rheol. 37, 695-726 (1993).

Baravian, C., D. Quemada, and A. Parker, "Modeling thixotropy using a novel structural kinetics approach. Basis and application to a solution of iota-carrageenan," J. Texture Stud. 27, 371-390 (1996).

Barnes, H. A., “Thixotropy—A review," J. Non-Newtonian Fluid Mech. 70, 1-33 (1997).

Bautista, F., J. M. de Santos, J. E. Puig, and O. Manero, "Understanding thixotropic and antithixotropic behavior of viscoelastic micellar solutions and liquid crystalline dispersions," J. Non-Newtonian Fluid Mech. 80, 93-113 (1999).

Brown, R., “Advances on thickener technology for waterborne coatings,” Eur. Polym. J. 184, 267-270 (1994).

Croll, S. G., and R. L. Kleinlein, "Influence of cellulose ethers on coatings performance," Adv. Chem. Ser. 213, 333-350 (1986).

Cross, M. M., "Rheology of non-Newtonian fluids. Flow equation for pseudoplastic systems," J. Colloid Sci. 20, 417-437 (1965).

De Kee, D., R. K. Code, and G. Turcotte, "Flow properties of time-dependent foodstuffs," J. Rheol. 27, 581-604 (1983).

Denny, D. A., and R. S. Brodkey, "Kinetic interpretation of non-Newtonian flow," J. Appl. Phys. 33, 22692274 (1962).

Emmons, W. D., and E. S. Travis, "Polyurethane thickeners in latex compositions," U.S. Patent No. 4079,028 (filed 1978).

Fredrickson, A. G., "A model for the thixotropy of suspensions," AIChE J. 16, 436-441 (1970).

Glass, J. E., D. N. Schulz, and C. F. Zukoski, "Polymers as rheology modifiers," ACS Symp. Ser. 462, 2-17 (1991).

Goodwin, J. W., and R. W. Hughes, "Particle interactions and dispersion rheology," ACS Symp. Ser. 663, 94-125 (1997).

Goodwin, J. W., R. W. Hughes, C. K. Lam, J. A. Miles, and B. C. H. Warren, "The rheological properties of a hydrophobically modified cellulose,” Adv. Chem. Ser. 223, 365-378 (1989). 
Groot, R. D., and G. M. Agterof, "Dynamic viscoelastic modulus of associative polymer networks: Off-lattice simulations, theory and comparison to experiments," Macromolecules 28, 6284-6293 (1995).

Hoy, K. L., and R. C. Hoy, "Polymers with hydrophobe branches," U.S. Patent No. 4,426,485 (filed 1984).

Jenkins, R. D., L. M. DeLong, and D. R. Basset, "Influence of alkali-soluble associative emulsion polymer architecture on rheology," Adv. Chem. Ser. 248, 425-447 (1996).

Jenkins, R. D., C. A. Silebi, and M. S. El-Aasser, "Steady-shear and linear-viscoelastic material properties of model associative polymer solutions," ACS Symp. Ser. 462, 222-233 (1991).

Kaczmarski, J. P., and J. E. Glass, "Synthesis and solution properties of hydrophobically-modified ethoxylated urethanes with variable oxyethylene spacer lengths," Macromolecules 26, 5149-5156 (1993).

Karlson, L., F. Joabson, and K. Thuresson, "Phase behavior and rheology in water and in model paint formulations thickened with HM-EHEC: Influence of the chemical structure and the distribution of hydrophobic tails," Carbohydr. Polym. 41, 25-35 (2000).

Karunasena, A., G. Brown, and J. E. Glass, "Hydrophobically modified ethoxylated urethane architecture. Importance for aqueous- and dispersed-phase properties," Adv. Chem. Ser. 223, 495-525 (1989).

Kästner, U., "The impact of rheological modifiers on water-borne coatings," Colloids Surf., A 183, 805-821 (2001).

Kästner, U., H. Hoffmann, R. Dönges, and R. Ehrler, "Interactions between modified hydroxyethyl cellulose (HEC) and surfactants," Colloids Surf., A 112, 209-225 (1996).

Kroon, G., "Associative behavior of hydrophobically modified hydroxyethyl celluloses (HMHECs) in waterborne coatings," Prog. Org. Coat. 22, 245-260 (1993).

Landoll, L. M., "Nonionic polymer surfactants," J. Polym. Sci. 20, 443-455 (1982).

Lapasin, R., M. Grassi, and S. Pricl, "Fractal approach to rheological modeling of aggregate suspensions," in A. Ait-Kadi, J. M. Dealy, D. F. James, and M. C. Williams, Proceedings of the XIIth International Congress on Rheology, Laval University, Quebec City, Canada, 1996, p. 524.

Maestro, A., C. González, and J. M. Gutiérrez, "Rheological behavior of HMHEC solutions," J. Rheol. 46, 127-143 (2002).

McCormick, C. L., and C. B. Johnson, "Synthetically structured water-soluble copolymers. Associations by hydrophobic or ionic mechanisms," Adv. Chem. Ser. 223, 437-454 (1989).

Mewis, J., “Thixotropy—A general review,” J. Non-Newtonian Fliud Mech. 6, 1-20 (1979).

Papir, Y. S., and I. M. Krieger, "Rheological studies on dispersions of uniform colloidal spheres. II. Dispersions in nonaqueous media," J. Colloid Interface Sci. 34, 126-130 (1970).

Quemada, D., "Rheological modeling of complex fluids. IV: Thixotropic and 'thixoelastic' behavior. Start-up and stress relaxation, creep tests and hysteresis cycles," Eur. Phys. J. A 5, 191-207 (1999).

Reuvers, A. J., "Control of rheology of water-borne paints using associative thickeners," Prog. Org. Coat. 35, 171-181 (1999).

Sau, A. C., and L. M. Landoll, "Synthesis and solution properties of hydrophobically modified (hydroxyethyl)cellulose," Adv. Chem. Ser. 223, 343-363 (1989).

Schwab, F. G., "Advantages and disadvantages of associative thickeners in coatings performance," Adv. Chem. Ser. 213, 369-373 (1986).

Seneker, S. D., and J. E. Glass, "Reaction parameter effects on substituent distributions in the heterogeneous synthesis of cellulose ethers," Adv. Chem. Ser. 248, 125-137 (1996).

Soong, D. S., and M. M. Shen, "A kinetic network model for nonlinear viscoelastic flow properties of entangled monodisperse polymers," J. Polym. Sci., Polym. Lett. Ed. 17, 595-599 (1979).

Svanholm, T., F. Molenaar, and A. Toussaint, "Associative thickeners: Their adsorption behavior onto latexes and the rheology of their solutions," Prog. Org. Coat. 30, 159-165 (1997).

Tam, K. C., R. D. Jenkins, M. A. Winnik, and D. R. Basset, "A structural model of hydrophobically modified urethane-ethoxylated (heur) associative polymers in shear flow," Macromolecules 31, 4149-4159 (1998).

Tanaka, R., J. Meadows, P. A. Williams, and G. O. Phillips, "Interactions of hydrophobically modified (hydroxyethyl)cellulose with various added surfactants," Macromolecules 25, 1304-1310 (1992).

Tarng, M. R., and J. E. Glass, "Thixotropy in HMHEC/SDS associative thickener aqueous solutions," Polym. Mater. Sci. Eng. 72, 380-381 (1995).

Tarng, M. R., M. Zeying, K. Alahapperuma, and J. E. Glass, "Associative thickeners in the land of commercial reality: Coating formulations,” Adv. Chem. Ser. 248, 450-486 (1996).

Tiu, C., and D. V. Boger, "Complete rheological characterization of time-dependent products," J. Texture Stud. 5, 329-338 (1974)

Vittadello, S. T., and S. Biggs, "Shear history effects in associative thickener solutions," Macromolecules 31, 7691-7697 (1998)

Wetzel, W. H., M. Chen, and J. E. Glass, “Associative thickeners. An overview with an emphasis on synthetic procedures," Adv. Chem. Ser. 248, 163-179 (1996).

Witten, T. A. and M. H. Cohen, "Cross-linking in shear-thickening ionomers," Macromolecules 18, 1915-1918 (1985).

Xu, B., A. Yekta, and M. A. Winnik, "Viscoelastic properties in water of comb associative polymers based on poly(ethylene oxide)," Langmuir 13, 6903-6911 (1997). 
Xu, B., A. Yekta, Z. Masoumi, and M. A. Winnik, "The functionality of associative polymer networks: The association behavior of hydrophobically modified urethane-ethoxylated (HEUR) associative polymers in aqueous solution," Colloids Surf., A 112, 239-250 (1996).

Yekta, A., J. Duhamel, H. Adiwidjaja, P. Brochard, and M. A. Winnik, "Fluorescence studies of associating polymers in water: Determination of the chain end aggregation number and a model for the association process," Macromolecules 28, 956-966 (1995).

Yekta, A., B. Xu, J. Duhamel, H. Adiwidjaja, and M. A. Winnik, “Association structure of telechelic associative thickeners in water," Langmuir 9, 881-883 (1993).

Zhang, L. M., "Cellulosic associative thickeners," Carbohydr. Polym. 45, 1-10 (2001). 
